\title{
Characterization of a Disease-causing Lys329 to Glu Mutation in 16 Patients with Medium- chain Acyl-CoA Dehydrogenase Deficiency
}

\author{
N. Gregersen ${ }^{1}$, B. S. Andresen ${ }^{1}$, P. Bross ${ }^{2}$, V. Winter ${ }^{1}$, N. RÜdiger ${ }^{1}$, \\ S. Engst ${ }^{2}$, S. Ghisla ${ }^{2}$, E. Christensen ${ }^{3}$, D. Kelly ${ }^{4}$, A. W. Strauss ${ }^{4}$, \\ S. KølvraA ${ }^{5}$, L. Bolund ${ }^{5}$, A. Blakemore ${ }^{6}$, D. Curtis ${ }^{6}$ and P. Engel ${ }^{6}$ \\ ${ }^{1}$ Molecular Genetic Laboratory, University Department of Clinical Chemistry, \\ Aarhus Kommunehospital and Skejby Sygehus, 8200 Aarhus N, Denmark; ${ }^{2}$ Faculty \\ of Biology, University of Konstanz, 7760 Konstanz, Germany; ${ }^{3}$ Section of Clinical \\ Genetics, University Department of Paediatrics and of Gynaecology and Obstetrics, \\ Rigshospitalet, 2100 Copenhagen $\emptyset$, Denmark; ${ }^{4}$ Department of Pediatrics, \\ Washington University School of Medicine, St Louis, Missouri 63110, USA; \\ ${ }^{5}$ Institute of Human Genetics, University of Aarhus, 8000 Aarhus C, Denmark; \\ ${ }^{6}$ Department of Molecular Biology and Biotechnology, The University of Sheffield, \\ Sheffield S10 2TN, UK
}

Medium-chain acyl-CoA dehydrogenase (MCAD) deficiency (McKusick 20145) is the most common inherited defect of the $\beta$-oxidation of fatty acid (Roe and Coates, 1989). It causes life-threatening attacks of hypoglycaemia and lethargy, and has led to sudden infant death in apparently asymptomatic children. It is therefore important to diagnose the enzyme deficiency in asymptomatic siblings of affected cases and in families with cases of sudden infant death syndrome and 'near miss'.

The existing enzymatic methods used for the diagnosis are all either non-specific or laborious, unsuited for use in routine laboratories. The interest in defining the molecular defect(s) at the DNA level has therefore - besides the obvious interest in gaining more insight into the disease - been to develop fast and easy diagnostic tests.

\section{MATERIALS AND METHODS}

Western and Northern blotting were performed by standard techniques, using porcine anti-MCAD antibody and a cDNA probe labelled with ${ }^{32} \mathrm{P}-\mathrm{dCTP}$, respectively (Kelly et al., 1987). The initial mutation-finding study was performed on polymerase chain reaction (PCR) produced cDNA from fibroblast RNA originating from a Danish family with one child suffering from MCAD deficiency (Kфlvraa et al., 1982). The detailed methods will be published elsewhere. The cDNA comprising the coding region for the mature MCAD polypeptide was cloned into pGEM4Z and sequenced by the dideoxy method. After identification of a common mutation, A to $G$, at 
position 985 in the cDNA, the mutation was tested in an E. coli expression system (Bross et al., 1990) to measure the activity of the mutant enzyme.

To analyse for the G985 mutation in genomic DNA in the family members, in other patients with MCAD deficiency and in control individuals, a genomic assay based on a PCR technique was developed. The sense primer (5'TTTATGCTGGCTGAAATGGCCATG) is located with its $3^{\prime}$-end at position 984 of the cDNA, creating an NcoI restriction site (CCATGG) when the mutant sequence is copied, but not when the normal sequence (CCATGA) is produced by PCR. The antisense primer (5'-CAGGATATTCTGTATTAAATCCATGGCCTC) creates another NcoI site, which functions as an internal control of the enzyme cleavage efficiency. The reaction conditions for the PCR were based on standard protocols. The assay is visualized in Figure 1. Haplotyping was performed on genomic DNA from the same individuals with BanII, PstI and TaqI restriction enzymes, using Southern blotting with a ${ }^{32}$ P-labelled MCAD cDNA as hybridization probe.

\section{RESULTS AND DISCUSSION}

Haplotyping using an MCAD cDNA probe was performed in order to check the inheritance in the family, to determine the genetic background(s) for the mutation(s) and to look for intragenic gross rearrangements, such as deletions. The analysis of the Danish family revealed that the patient (RB) was homozygous for haplotype 112, using respectively BanII, PstI and TaqI restriction endonucleases, and that the

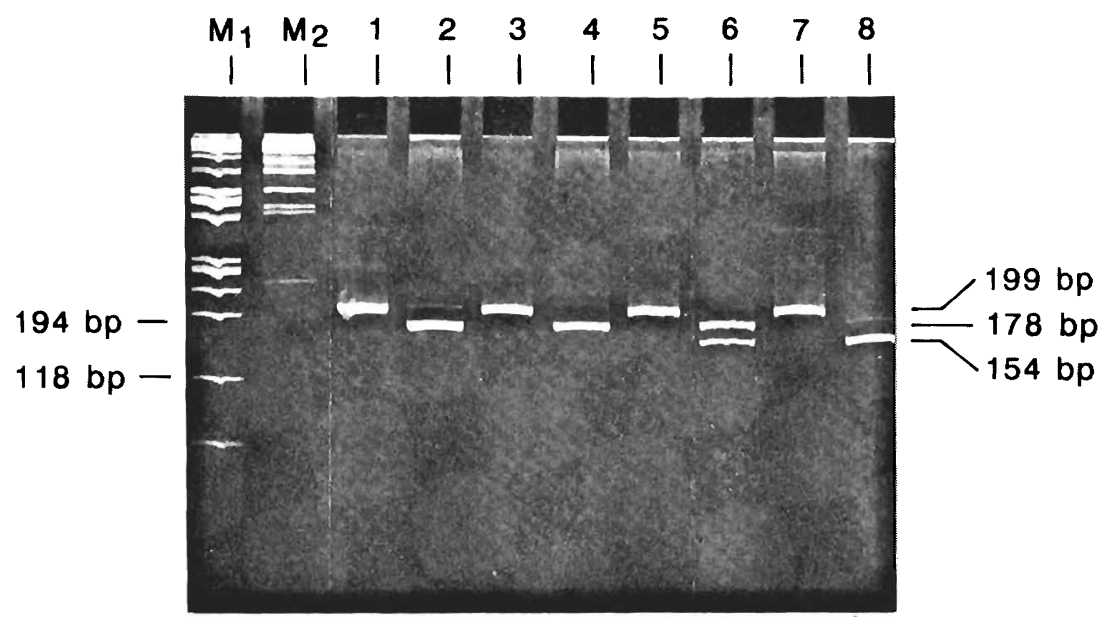

Figure 1 Ethidium bromide stained polyacrylamide gel (PAGE) showing the genomic analysis for G985 in a patient, a heterozygous individual and two control individuals. PCR products were either loaded directly (lanes with uneven numbers), or digested with NcoI before electrophoresis (lanes with even numbers). Lane $\mathbf{M}_{1}$ : size marker (mixture of HindIII digested lambda $\mathrm{C}_{1} 857$ DNA and HaeIII digested $\Phi$ X-174 DNA). Lane $\mathbf{M}_{2}$ : size marker. Lanes 1 and 2: control. Lanes 3 and 4: control. Lanes 5 and 6: individual heterozygous for G985. Lanes 7 and 8: patient with MCAD deficiency homozygous for G985 
obligate heterozygous parents were heterozygous for the same haplotype. This haplotype occurs in approximately $25 \%$ of normal alleles. Furthermore, no abnormal bands occurred compared to normals, indicating strongly that both alleles posses the normal amount and sizes of exons and introns.

These results together with the presence of protein and mRNA of a size indistinguishable from controls pointed very strongly to point mutations in the coding region for the mature polypeptide as being the cause of the disease. For this reason primers for PCR amplification of CDNA, isolated from fibroblast RNA, were designed with cloning sites in the 5 '-end of each primer, enabling the PCR product to be easily cloned into the polylinker of pGEM4Z. Since the Taq-polymerase in the PCR reaction may misincorporate mononucleotides at a rate of $10^{-4}$, several clones from each cDNA were sequenced. The analysis revealed as the only consistent mutation compared to the normal sequence a base-shift from $A$ to $G$ at position 985 of the MCAD cDNA. Sequence analysis of cDNA clones from the parents revealed both A985 and G985, consistent with their heterozygous status.

The mutation changes a lysine to glutamic acid in the MCAD protein. Insertion of the mutant sequence into an expression vector followed by expression in $E$. coli produced an enzyme with decreased activity. This and the fact that G985 was not observed in genomic DNA from 30 control individuals proved G985 to be the diseasecausing mutation in this family.

By means of the very easy genomic assay for G985 it was found that 15 out of 16 patients with MCAD deficiency showed homozygosity for G985. The 16th was heterozygous for the mutation, apparently possessing another disease-causing mutation in addition to G985. Haplotyping of these same 16 patients revealed that the same 15 who were homozygous for G985 were also homozygous for the haplotype 112. Because of lack of DNA from the parents of the 16th patient the haplotype cannot be resolved. However, the strong association between G985 and haplotype 112 suggests a founder effect.

\section{REFERENCES}

Bross, P., Engst, S., Strauss, A. W., Kelly, D. P., Rasched, I. and Ghisla, S. Characterization of wild-type and an active site mutant of human medium-chain acyl-CoA dehydrogenase after expression in E. coli. J. Biol. Chem. 265 (1990) 7116-7119

Kelly, D. P., Kim, J. J., Billadello, J. J., Hainline, B. E., Chu, T. W. and Strauss, A. W. Nucleotide sequence of medium-chain acyl-CoA dehydrogenase mRNA and its expression in enzyme-deficient human tissue. Proc. Natl. Acad. Sci. USA 84 (1987) 4068-4072

K $\phi$ lvraa, S., Gregersen, N., Christensen, E. and Hobolth, N. In vitro fibroblast studies in a patient with $\mathrm{C}_{6}-\mathrm{C}_{10}$-dicarboxylic aciduria: Evidence for a defect in general acyl-CoA dehydrogenase. Clin. Chim. Acta 126 (1982) 53-67

Roe, C. R. and Coates, P. M. Acyl-CoA dehydrogenase deficiencies. In: Scriver, C. R., Beaudet, A. L., Sly, W. S., Valle, D. (eds.), The Metabolic Basis of Inherited Disease, McGraw-Hill, New York, 1989, pp. 889-914 